\title{
Suture-Based Debris Behavior in the Draining Lymph Nodes of a Porcine Knee: A Study of Silicone, Polyethylene and Carbon
}

\author{
F. Alan Barber, M.D., F.A.C.S., David B. Spenciner, P.E., Sc.M., M.B.A., Brett G. Zani, Ph.D., \\ and Raffaele Melidone, D.V.M., D.A.C.V.P.
}

\begin{abstract}
Purpose: To determine whether debris from a silicone core suture has an observable intra-articular or extra-articular impact or can be shown to migrate into the lymphatic system. Methods: Using a porcine stifle joint model, 2 study groups were created: 1 group used silicone-suture particles created by rupturing hand-tied knots of a nonabsorbable suture with an outer sheath of ultrahigh molecular-weight polyethylene, an inner polyester sheath and a medical-grade silicone/sodium chloride-filled core. The second group used a mixture of 3 vitreous carbon particles sizes. Twelve Yorkshire pigs were randomly assigned to each group. Only l type of study particle was used in a single animal to avoid crosscontamination. Half of the study materials were placed by arthrotomy into the joint and, after capsular closure, the remaining half were placed on the joint capsule before skin closure. Six weeks postimplantation, the stifle joints and regional lymph nodes were examined macroscopically and microscopically. The extracapsular and intra-articular tissue and the lymph nodes were examined. Results: Macroscopically, none of the 12 silicone-suture particle group limbs had abnormal macroscopic observations. Of the 12 limbs in the carbon particle group, 8 demonstrated discoloration in the extracapsular, intra-articular and lymph node tissues; 6 limbs demonstrated joint discoloration (extracapsular and intraarticular). The silicone-suture particle group showed lymph node inflammation in $25 \%$ of the iliac lymph nodes and $42 \%$ of the inguinal lymph nodes but none in the popliteal lymph nodes. In the carbon particle group, 100\% of the iliac lymph nodes, $75 \%$ of the inguinal lymph nodes and $8 \%$ of the popliteal nodes showed inflammation. No silicone-suture debris migration was observed microscopically. Visible carbon particle migration was present in $100 \%$ of the iliac lymph nodes and in $50 \%$ of the inguinal lymph nodes but in none $(0)$ of the popliteal lymph nodes. The carbon particle group also showed inflammation in these areas. Conclusions: Carbon particles in the porcine knee migrated into the lymph nodes (iliac 100\%; inguinal 50\%, popliteal 0); gross discoloration was observed in 8 of 12 specimens. Silicone-suture particles could not be found in the lymph nodes, and no macroscopic joint damage was observed. Clinical Relevance: The clinical use of a suture containing a silicone/salt central core is common, and the porcine lymphatic model suggests that this type of suture material does not result in clinically significant silicone particulates.
\end{abstract}

$\boldsymbol{S}^{\text {o }}$ oft-tissue repair, including the rotator cuff tendons, requires approximation of the tendon to bone in a stable manner throughout the healing period. The weakest link of this repair is the tendon-suture

From the Plano Orthopedic Sports Medicine and Spine Center (F.A.B.), Plano, Texas; DePuy Synthes Mitek Sports Medicine (D.B.S.), Raynham, Massachusetts; and Concord Biomedical Sciences and Emerging Technologies (B.G.Z., R.M.), Lexington, Massachusetts, U.S.A.

The authors report the following potential conflicts of interest or sources of funding: This research was funded by a grant from DePuy Synthes Mitek Sports Medicine, Raynham MA. F.A.B. has received support in the form of grants from DePuy-Mitek, and support for travel to meetings for the study or other purposes from DePuy-Mitek, and has received support from DePuyMitek for grants or grants pending, consultancy, lectures including service on speakers bureaus, and royalties, outside the submitted work; D.B.S. is an employee of Johnson $\theta$ Johnson, which developed the test suture used in this study; and B.G.Z. and R.M. report that Concord Biomedical Sciences and interface. $^{1,2}$ Causes of repair failure include the lack of consistent tendon approximation to bone from knot rupture, knot slippage ${ }^{3,4}$ and suture loosening, ${ }^{5}$ which can be magnified by patient noncompliance during the

\footnotetext{
Emerging Technologies is a non-profit biomedical research institute contracted to conduct the animal study. Full ICMJE author disclosure forms are available for this article online, as supplementary material.

Received September 13, 2019; accepted September 17, 2019.

Address correspondence to F. Alan Barber, M.D., F.A.C.S., Plano Orthopedic Sports Medicine and Spine Center, 800 Broadmoor Lane, Propser, TX 75078,U.S.A.E-mail: knees2do@gmail.com

(C) 2019 by the Arthroscopy Association of North America. Published by Elsevier Inc. This is an open access article under the CC BY-NC-ND license (http://creativecommons.org/licenses/by-nc-nd/4.0/).

2666-061X/191120

https://doi.org/10.1016/j.asmr.2019.09.006
} 
healing period. The viscoelastic property creep affects suture material, and suture loosening can be observed shortly after a repair construct has been completed. ${ }^{6}$ Suture loosening after rotator cuff repair using a knotless bridging technique was clinically described by Hayeri et al. and resulted in subacromial-subdeltoid bursitis. $^{7}$ In vitro biomechanical testing demonstrates that suture-holding strength with some knotless suture anchors is lower than the associated anchor's pullout strength. $^{8}$ Suture loosening with transosseousequivalent suture-bridge repairs can affect the footprint area, the pressure at the tendon-bone contact site $^{9,10}$ and, in knotless anchor systems, can result in the failure of the tested constructs to reach half the anchor pullout strength achieved prior to suture slippage. ${ }^{3}$

A nonabsorbable ultrahigh molecular-weight polyethylene (UHMWPE)-containing suture with an inner core of silicone $/ \mathrm{NaCl}$ that was released recently may address this creep issue. When this suture is placed in an aqueous environment without tension, the salt particles in the silicone core elute out, leaving a microporous structure in the core. These small voids are subsequently filled by the surrounding fluid, resulting in radial expansion and axial shortening of the core. This mechanical effect may reverse some of the negative creep effects. The practical clinical implication of this process is reduced suture loosening. However, concerns exist that the silicone core could shed clinically significant debris during either suture tying or if suture rupture occurs. In a pilot study using this suture, knots were tied and then broken. A single ruptured knot produced more than 200 particles, ranging from less than $10 \mu \mathrm{m}$ to more than $100 \mu \mathrm{m}$ in size. The purpose of this study was to determine whether debris from a silicone core suture has an observable intraarticular or extra-articular impact or can be shown to migrate into the lymphatic system. The study hypothesis was that if the silicone core of this UHMWPE suture ruptures, detectable silicone particles will not migrate into the lymphatic system or cause observable damage.

\section{Methods}

This study was performed at an independent testing laboratory in compliance with Food and Drug Administration Good Laboratory Practices. The test protocol was approved by the appropriate Institutional Animal Care and Use Committee and was consistent with a previously validated methodology (blinded). Qualityassurance monitors were conducted during in-process inspections at critical phases of the study, including but not limited to material implantation and material recovery. The study was designed to represent a worstcase scenario in which multiple knots were forcibly ruptured at the same time. Such rupturing could potentially result in the release of suture and silicone particulates into the joint or the surgical area without the benefit of any encapsulating tissue sleeve such as that which forms around all nonabsorbable sutures soon after implantation.

A porcine model was chosen because of its similarities to the human joint. ${ }^{11,12}$ For this study, 12 adolescent male Yorkshire pigs (Sus scofa domesticus) weighing 40$50 \mathrm{~kg}$ were obtained. All animals were placed in quarantine and subsequently underwent a standard veterinary examination to confirm their healthy status before inclusion in the study. None of the 12 was rejected. The pigs were randomly assigned by using a computer-generated matrix of 4 secured in sealed envelopes, which were opened at the time of implantation. There were 2 groups, and both knees of each animal were used for implantation. The study group included 6 animals ( 12 joints) that received the study's suture particles, and 6 animals ( 12 joints) were assigned to the control group and received vitreous carbon particles. Both knees in each study animal received the same material to avoid cross-contamination within the lymphatic system. The material was introduced into the joint and, after joint capsule closure, into the extracapsular space. The 12 knees in the control group were implanted with a mixture of differently sized vitreous carbon particles. The 12 knees in the study group were implanted with the silicone-suture particles, which were created as follows.

The study material was a \#2 high-strength suture (braided UHMWPE surrounding an inner braided polyester sheath and a medical-grade silicone/sodium chloride-filled central score) (DYNACORD suture, DePuy Synthes, Raynham, Massachusetts). Using lengths of this ethylene oxide sterilized \#2 suture, 375 Duncan loop knots backed up with 2 alternating half hitches were tied around a $10 \mathrm{~mm}$ mandrel. These knots were tied using a special knot pusher with a load cell attached to it. The load cell allowed the tying of consistently tensioned knots. The force on each half hitch, when tied, was $112 \mathrm{~N}$. Thirty pretied knots were used for each knee. The knots were mechanically ruptured using sterilized snap ring pliers inside the pig joints, which had been surgically opened by a limited arthrotomy. In a pilot study, it was determined via manual counting with microscopic imaging (under 100x magnification) that the silicone debris generated by each knot rupture with this methodology averaged 27 particles less than $10 \mu \mathrm{m}$ in the longest dimension, 133 particles between 10 and $25 \mu \mathrm{m}, 39$ particles between 25 and $50 \mu \mathrm{m}, 39$ particles between 50 and 100 $\mu \mathrm{m}$, and 1 particle larger than $100 \mu \mathrm{m}$. After each knot rupture, the remnant suture was removed from the surgical field. Two insertion sites were dosed with the created silicone-suture debris from 15 ruptured knots each: the intra-articular space and, after a water-tight closure of the joint capsule, the extra-capsular space. 


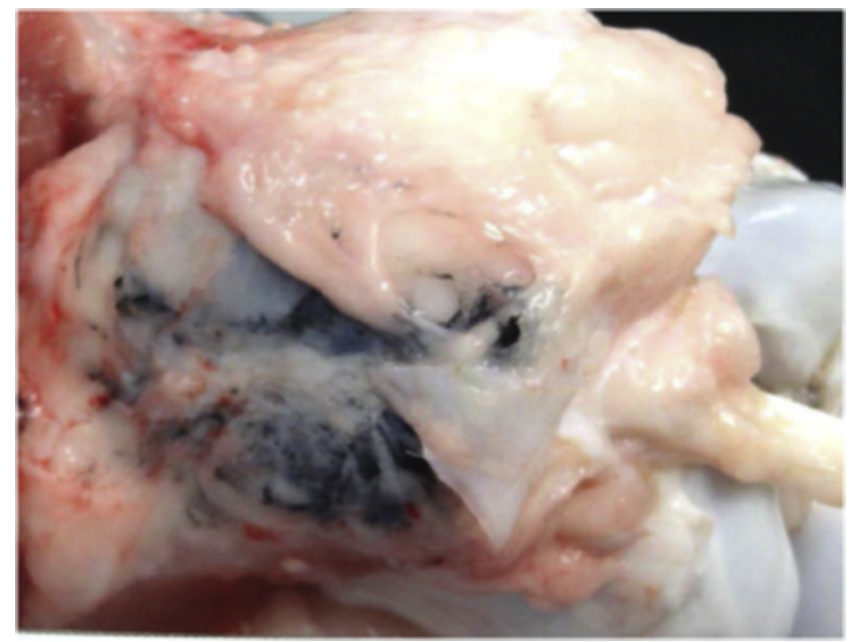

Fig 1. Right stifle joint extracapsular tissue reveals carbon particles imbedded in the tissue. (Copyright by F. Alan Barber M.D., F.A.C.S.)

Afterward, the wounds were closed routinely. There were 15 unused knots.

The control group was implanted with vitreous carbon particles. Carbon fibers are commonly combined with other materials, including polyether ether ketone, for use in orthopedic applications. ${ }^{13,14}$ Carbon debris has been shown to produce synovitis and nonspecific foreign-body reactions, but they are not of sufficient severity or duration to alter the mechanical properties of knee cartilage. ${ }^{13,15}$ The vitreous carbon particles were prepared by mixing $0.06 \mathrm{~g}$ of Goodfellow Vitreous Carbon (VC006010/6, sized $0.4 \mu \mathrm{m}$ to $12 \mu \mathrm{m}$ ) powder, $0.45 \mathrm{~g}$ of Goodfellow Vitreous Carbon (VC006015/2, sized 20 $\mu \mathrm{m}$ to $50 \mu \mathrm{m}$ ) powder, $0.81 \mathrm{~g}$ of Goodfellow Vitreous Carbon (VC006021/1, sized less than $300 \mu \mathrm{m}$ ) powder, and approximately $10 \mathrm{~mL}$ of sterile saline. This mixture was sterilized prior to insertion. The resulting sterile solution was shaken immediately prior to insertion, and then half of the solution was introduced into the joint through the arthrotomy wound. After the capsule was closed, the remainder of the solution was placed onto the knee capsule. The wound was then closed.

\section{Surgical Procedure}

The animals were fasted prior to the implantation surgery and were premedicated and anesthetized according the standard animal facility protocol approved by the veterinarian and study director.

A general intubation anesthesia was used, including a peripheral IV catheter for administration of supportive IV fluids. Accepted veterinary care standards were followed. The incision sites were aseptically prepared and draped for the procedure. A parapatellar incision was made and carried down to the capsule. A capsulotomy was performed and the patellar retracted. Each animal received only 1 type of material. The postoperative care for all animals was the same.

Six weeks after the implantation procedures, the material was examined. The animals were returned to the facility and euthanized. A peripheral IV catheter was placed, and each animal was euthanized under anesthesia in accordance with accepted American Veterinary Medical Association guidelines. The animals were administered tiletamine hydrochloride and zolazepam hydrochloride and anesthetized via isoflurane inhalant.

Immediately after euthanasia, the knees and the appropriate lymph nodes were harvested. The prior surgical incision in both knees, with the underlying tissue, was excised en bloc and examined for visible extracapsular debris (particulates). This included the skin, the subcutaneous tissue and the joint capsule. The were later inspected microscopically to determine whether residual study or control material was present in the implantation sites.

The patellar tendon was detached from the tibia and reflected proximally to expose the joint fully. The joint was macroscopically examined for visible debris and any indication of an inflammatory reaction. Articular and meniscal cartilage damage, if present, was noted. Inflammatory synovitis, deposits in the fat pad or other intra-articular abnormalities were noted. The specimens and the joint contents were documented photographically and harvested for histology. Additionally, right and left surface inguinal, popliteal and iliac lymph nodes were harvested, visually examined and prepared

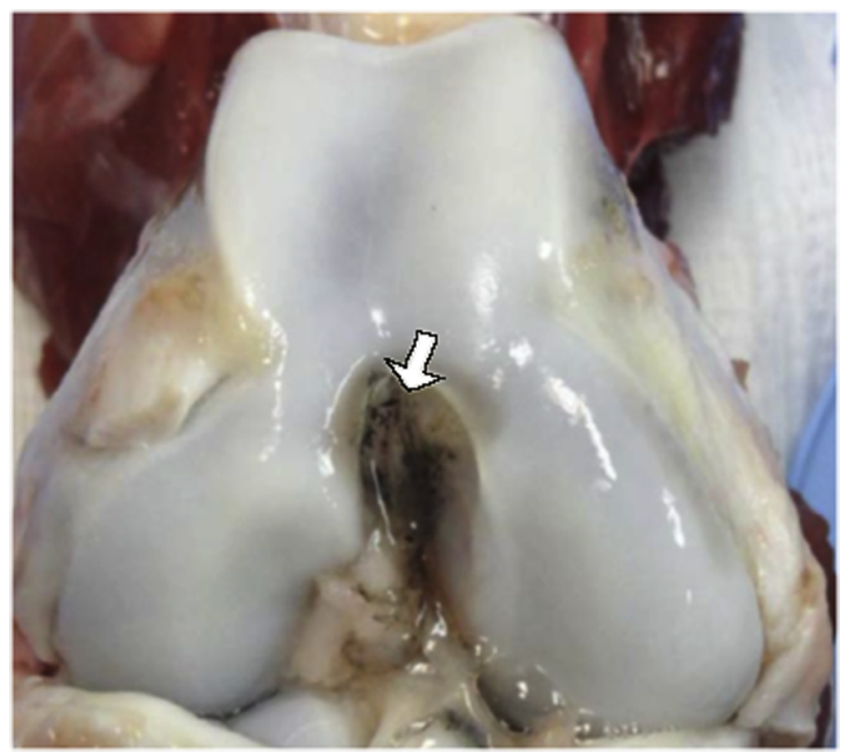

Fig 2. Right stifle anterior joint view reveals articular cartilage and cruciate ligaments with carbon particles (white arrow) imbedded in the synovial tissue in the intracondylar notch but no articular cartilage abrasion. (Copyright by F. Alan Barber M.D., F.A.C.S.) 


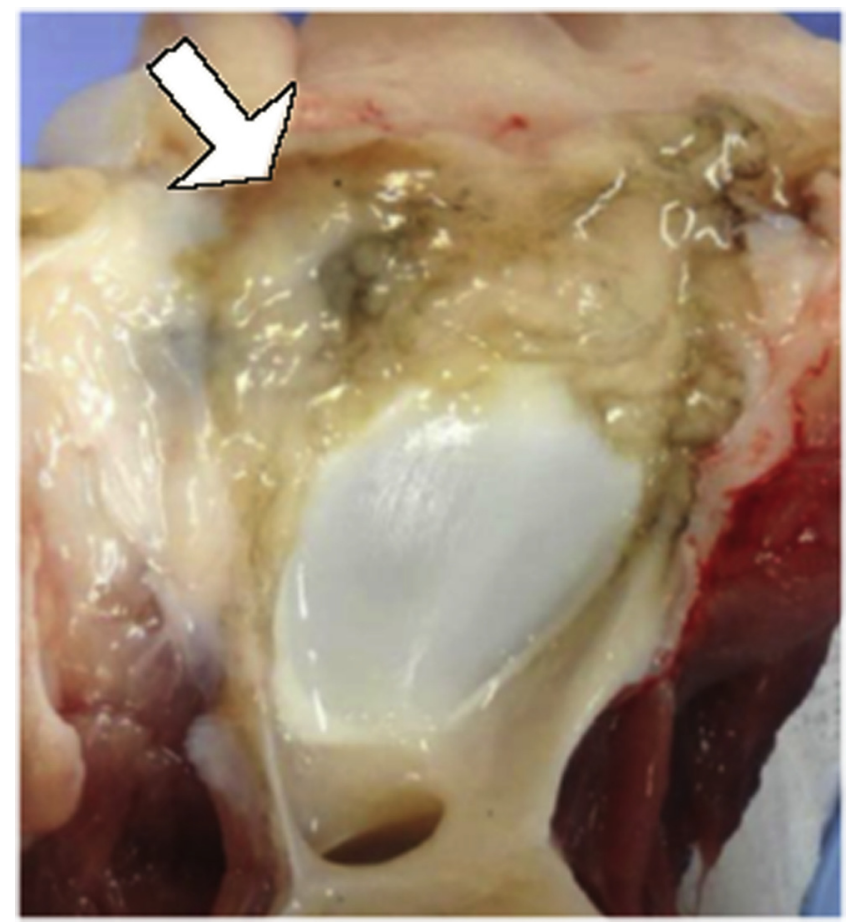

Fig 3. Right stifle joint reflected patella demonstrates brown carbon staining (white arrow) of the synovium surrounding the patella but no articular cartilage damage. (Copyright by F. Alan Barber M.D., F.A.C.S.)

for histologic examination (6 total lymph nodes per animal).

The harvested lymph nodes were bisected; the cut faces were placed side-by-side and embedded in paraffin. In this manner, each slide incorporated 2 sections of tissue. Slides were approximately $0.6 \mathrm{~mm}$ apart in the block and were stained with hematoxylin and eosin. Tissues with macroscopic changes were paraffin processed and stained with hematoxylin and eosin for histomorphologic assessment.

Light microscopy was used for characterization of the host responses, and they were evaluated for foreign material, by using standard illumination and polarized light, for the presence of inflammation and inflammatory cell subtypes, any disruption of tissue architecture and signs of tissue injury, such as cell injury and death. The study pathologist was blinded to the treatment groups at the time of the evaluation.

The study's endpoints included the presence of visible debris in the various sites (extra-capsular, intraarticular, lymph nodes), the presence of visible changes in the joint and the presence of microscopic debris in the lymph nodes.

\section{Statistical Evaluation}

A sample size calculation was performed a priori. It was determined that a sample size of 10 knees would provide a power of 0.8 and a confidence of $95 \%$. Values and observations obtained from the histomorphologic analysis were entered into a Microsoft Excel spreadsheet. Ordinal histologic data (scores) were reported as the group median, mean \pm standard deviation and percent incidence, as appropriate. For the binary data addressing the presence or absence of particulate in the lymph nodes, statistical software (Minitab 17, Mintab, State College, Pennsylvania), a 2 proportion test was used to calculate the differences between the 2 groups. Statistical significance was set at 0.05 .

\section{Results}

All 12 animals survived to the 6-week time point. They were all healthy and gained weight throughout the study and had no changes in ambulatory status. Some transient inguinal lymph node enlargement was noted. However, all lymph nodes were within normal limits prior to necropsy. For 1 of the animals in the test group, the popliteal lymph nodes were absent bilaterally.

The presence of visible debris in the extracapsular area, the intra-articular area and the lymph nodes was evaluated. In the suture particle group, 11 of the 12 limbs showed no abnormal observations. One left leg demonstrated a brown/yellow viscous fluid accumulation in the joint, but this had been documented on day 0 , prior to particle insertion, and was considered an incidental finding. Consequently, it was found that no evidence existed of any visible discoloration or damage occurred in association with the suture particles.

The carbon-particle group demonstrated gross discoloration (dark to black) in 8 of the 12 limbs. This discoloration occurred in the extracapsular (Fig 1), intra-articular (Fig 2) and lymph node tissues. Two different animals demonstrated gross discoloration in

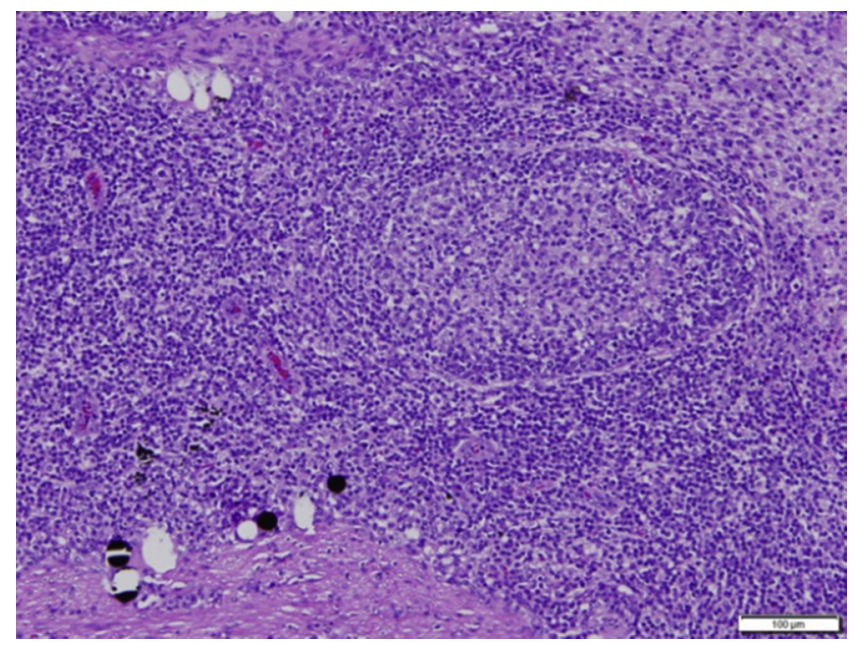

Fig 4. Hematoxylin and eosin stained 10x power view of a left iliac lymph node showing carbon particles (black) and white spaces left empty by the carbon particles, which were removed during processing. (Copyright by F. Alan Barber M.D., F.A.C.S.) 


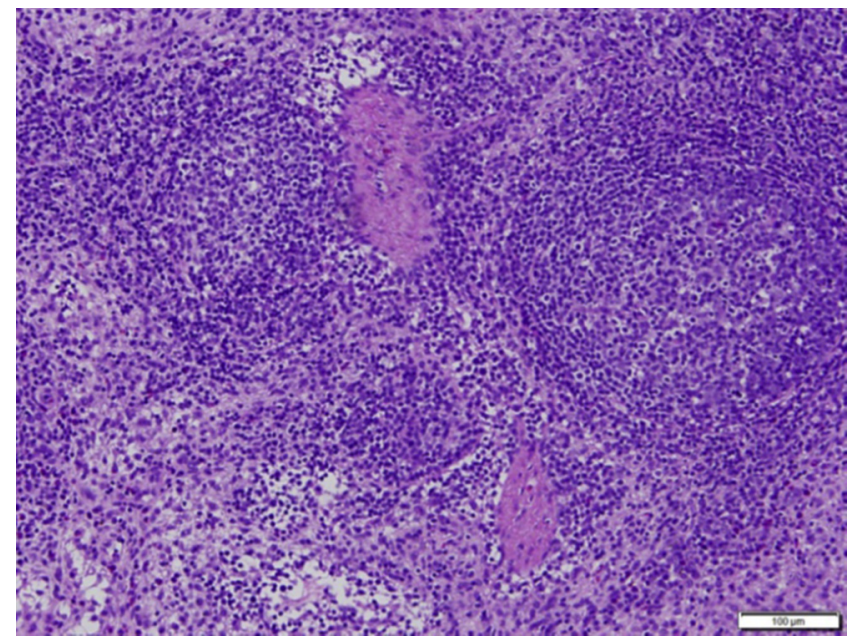

Fig 5. Hematoxylin and eosin (H\&E)-stained 10x power view of a left iliac lymph node from the suture particle group without any foreign material particles. (Copyright by F. Alan Barber M.D., F.A.C.S.)

the lymph nodes (right iliac and left popliteal lymph nodes). Three animals demonstrated subcutaneous discoloration in both knees in extracapsular (Fig 3) and intra-articular sites (6 knees).

No observable abrasion damage was found in the articular cartilage or other intra-articular or extracapsular tissues of any specimen (Figs 1-3). However, microscopically, inflammation in the suture-particle group was present in $25 \%$ of the iliac lymph nodes and $42 \%$ of the inguinal lymph nodes but absent in the popliteal lymph nodes. Inflammation in the carbon particle group was present in $100 \%$ of the iliac lymph nodes, $75 \%$ of the inguinal lymph nodes and $8 \%$ of the popliteal nodes. Visible migration of carbon was present in $100 \%$ of the iliac lymph nodes (Fig 4) and $50 \%$ of the inguinal lymph nodes but in none $(0)$ of the popliteal lymph nodes. No silicone debris or foreign-body reactions were present in any lymph node (Fig 5). The differences in incidence of particulates was statistically significantly higher for the carbon particles in the iliac $(P<0.001)$ and inguinal $(P=$ 0.014) lymph nodes.

\section{Discussion}

Carbon particles placed in the knee migrated into the iliac lymph nodes $100 \%$ of the time and into the inguinal lymph nodes $50 \%$ of the time but did not migrate into the popliteal nodes (0). Silicone suture particles could not be found in any lymph node. Extracapsular, intra-articular or lymph node gross discoloration was present in 8 of the 12 limbs in the carbon-particle group but in none of the silicone-suture particle group. Observable macroscopic damage to the articular cartilage was not observed in either group.
Metallic or polyethylene particle migration is common after joint replacement and in other clinical settings. ${ }^{16-20}$ Silicone has also been reported to migrate. ${ }^{21-24}$ Synthetic ligaments have demonstrated wear particles and fibers in the joint space and lymph nodes at 1 year. ${ }^{17}$ The regional lymph nodes (inguinal, iliac and para-aortic) were commonly involved, including the contralateral limb nodes. Although the use of a silicone core suture in arthroscopic rotator cuff tendon repair may raise safety concerns, the presence of silicone in suture material is not new. Another highstrength suture has a surface silicone coat, but reported adverse events such as granulomas related to the silicone are rare in the literature. ${ }^{6}$ The current study failed to demonstrate any silicone particle migration into the surrounding tissue or the lymphatic system.

The absence of evidence of silicone or suture particulate migration does not eliminate the possibility of its occurring, but macroscopic evidence was clearly absent, and a thorough microscopic evaluation failed to reveal it. In contrast, the control carbon particles were readily visible. A suture that can minimize laxity and counter subsequent repair elongation is attractive and is gaining widespread use. It is important to obtain as much data as possible regarding its safety.

\section{Limitations}

Study limitations include the use of a porcine instead of a human model. Only a 6 -week time point was evaluated. It is possible that some particles had already cleared the lymph nodes by the time of examination or that other particles might have taken longer to reach them. The results from this animal model cannot be directly applied to clinical experience. This study applies only to carbon, silicone and UHMWPE particles and cannot be extrapolated to other materials.

\section{Conclusions}

Carbon particles in the porcine knee migrated into the lymph nodes (iliac 100\%, inguinal 50\%, popliteal 0); gross discoloration was observed in 8 of 12 specimens. Silicone particles could not be found in the lymph nodes, and no macroscopic joint damage was observed.

\section{Acnowledgments}

This research was funded by a grant from DePuy Synthes Mitek Sports Medicine, Raynham MA.

\section{References}

1. Ito R, Suami H. Lymphatic territories (lymphosomes) in swine: An animal model for future lymphatic research. Plast Reconstr Surg 2015;136:297-304.

2. Kuptniratsaikul S, Weerawit P, Kongrukgreatiyos K, Promsang T. Biomechanical comparison of four sliding knots and three high-strength sutures: Loop security is 
much different between each combination. J Orthop Res 2016;34:1804-1807.

3. Wieser K, Farshad M, Vlachopoulos L, et al. Suture slippage in knotless suture anchors as a potential failure mechanism in rotator cuff repair. Arthroscopy 2012;28: 1622-1627.

4. Clark RR, Dierckman B, Sampatacos N, Snyder S. Biomechanical performance of traditional arthroscopic knots versus slippage-proof knots. Arthroscopy 2013;29: 1175-1181.

5. Klink CD, Binnebosel M, Alizai HP, et al. Tension of knotted surgical sutures shows tissue-specific rapid loss in a rodent model. BMC Surg 2011;11:36.

6. Ollivere BJ, Bosman HA, Bearcroft PW, Robinson AH. Foreign body granulomatous reaction associated with polyethelene Fiberwire suture material used in Achilles tendon repair. Foot Ankle Surg 2014;20:e27-e29.

7. Hayeri MR, Keefe DT, Chang EY. Suture slippage in knotless suture anchors resulting in subacromialsubdeltoid bursitis. Skel Radiol 2016;45:703-706.

8. Barber FA, Bava ED, Spenciner DB, Piccirillo J. Cyclic biomechanical testing of biocomposite lateral row knotless anchors in a human cadaveric model. Arthroscopy 2013;29: 1012-1018.

9. Zheng N, Harris HW, Andrews JR. Failure analysis of rotator cuff repair: A comparison of three double-row techniques. J Bone Joint Surg Am 2008;90:1034-1042.

10. Kummer F, Hergan DJ, Thut DC, Pahk B, Jazrawi LM. Suture loosening and its effect on tendon fixation in knotless double-row rotator cuff repairs. Arthroscopy 2011;27:1478-1484.

11. Martin RK, Gillis D, Leiter J, Shantz JS, MacDonald P. A porcine knee model Is valid for use in the evaluation of arthroscopic skills: A pilot study. Clin Orthop Relat Res 2016;474:965-970.

12. Proffen BL, McElfresh M, Fleming BC, Murray MM. A comparative anatomical study of the human knee and six animal species. Knee 2012;19:493-499.

13. Jockisch KA, Brown SA, Bauer TW, Merritt K. Biological response to chopped-carbon-fiber-reinforced peek. J Biomed Mater Res 1992;26:133-146.
14. Steinberg EL, Rath E, Shlaifer A, et al. Carbon fiber reinforced PEEK Optima: A composite material biomechanical properties and wear/debris characteristics of CFPEEK composites for orthopedic trauma implants. J Mech Behav Biomed Mater 2013;17:221-228.

15. Parsons JR, Bhayani S, Alexander H, Weiss AB. Carbon fiber debris within the synovial joint: A time-dependent mechanical and histologic study. Clin Orthop Relat Res 1985; 196:69-76.

16. Urban RM, Jacobs JJ, Tomlinson MJ, et al. Dissemination of wear particles to the liver, spleen, and abdominal lymph nodes of patients with hip or knee replacement. J Bone Joint Surg Am 2000;82:457-476.

17. Margevicius KJ, Claes LE, Durselen L, Hanselmann K. Identification and distribution of synthetic ligament wear particles in sheep. J Biomed Mater Res 1996;31: 319-328.

18. Lalor PA, Namba R, Mitchell SL, et al. Migration of polyethylene particles around stable implants in an animal model. J Long Term Eff Med Implants 1999;9:261-272.

19. Lemperle G, Morhenn VB, Pestonjamasp V, Gallo RL. Migration studies and histology of injectable microspheres of different sizes in mice. Plast Reconstr Surg 2004;113: 1380-1390.

20. Utzschneider S, Lorber V, Dedic M, et al. Biological activity and migration of wear particles in the knee joint: An in vivo comparison of six different polyethylene materials. J Mater Sci Mater Med 2014;25:1599-1612.

21. Henly DR, Barrett DM, Weiland TL, et al. Particulate silicone for use in periurethral injections: Local tissue effects and search for migration. J Urol 1995;153:2039-2043.

22. Leong AS, Disney AP, Gove DW. Spallation and migration of silicone from blood-pump tubing in patients on hemodialysis. N Engl J Med 1982;306:135-140.

23. Barrett DM, O'Sullivan DC, Malizia AA, Reiman HM, Abell-Aleff PC. Particle shedding and migration from silicone genitourinary prosthetic devices. J Urol 1991;146: 319-322.

24. Tehrani H, Lamberty BG. Axillary lymphadenopathy secondary to tattoo pigment and silicone migration. J Plast Reconstr Aesthet Surg 2008;61:1381. 